\title{
Grüner produzieren in Lateinamerika
}

\author{
Auch der Mercosur setzt auf die wirtschaftlichen Vorteile der Integration. Um- \\ weltpolitik und nachhaltige Entwicklung spielen dabei bislang noch eine unter- \\ geordnete Rolle, auch wenn die nachhaltige Nutzung der Ressourcen Wohlstand \\ sichert und eine Orientierung auf umweltverträgliche Produkte heute oft Markt- \\ vorteile bedeutet. Die Technische Zusammenarbeit trägt erfolgreich dazu bei, \\ den vermeintlichen Gegensatz zwischen Wettbewerbsfähigkeit und Umwelt zu \\ überwinden.
}

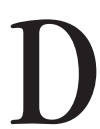
Von Detlef Schreiber er Mercado Común del Sur (Mercosur zu Deutsch Gemeinsamer Markt des Südens) wurde 1991 von Argentinien, Brasilien, Paraguay und Uruguay gegründet und erhielt 1994 mit dem Protokoll von Ouro Preto seine institutionelle Struktur. Er galt zumindest vor den schweren Wirtschaftskrisen, die die Region Ende der 90er Jahre und noch heftiger 2001/2002 erfassten, als erfolgreichstes Integrationsprojekt des Südens.

Nach einem krisenbedingten Erlahmen der Integrationsdynamik und Konflikten aufgrund unterschiedlicher Wechselkursregime erfährt der Mercosur heute wieder krättige Impulse, die aus politischen Übereinstimmungen zwischen den derzeitigen Regierungen resultieren. Diese Bewegung reicht heute weit über die vier Mitglieder des Mercosur hinaus: Im Dezember 2004 wurde die südamerikanische Staatengemeinschaft (Comunidad Sudamericana de Naciones) gegründet. Zudem traten dem Mercosur nach Chile, Bolivien und Peru nun auch Ecuador, Kolumbien und Venezuela als assoziierte Mitglieder bei. Das Bemühen um regionalen Schulterschluss ist auch vor dem Hintergrund diverser Handelsabkommen zu verstehen. Gemeinsam hat man eine stärkere Position. Dies darf aber nicht darüber hinweg täuschen, dass der Mercosur durch eine Reihe von internen Ausnahmeregelungen sowie Schwächen in der institutionellen Verankerung nicht so stark ist, wie es scheint.

\section{- Umweltpolitik und nachhaltige Entwicklung}

Ohne Zweifel sind die treibenden Kräfte der regionalen Integration des Mercosur wirtschaftsund handelspolitischer Natur. Allerdings führte der sukzessive Abbau von Zollschranken zu einem verstärkten Binnenhandel und einem wirtschaftlichen Strukturwandel, dessen soziale und ökologische Folgen zunehmend sichtbar werden. Auch die geplanten Projekte zum Ausbau der Infrastruktur sind von hoher Umweltrelevanz und werden von einer interessierten Öffentlichkeit aufmerksam verfolgt, der bewusst ist, dass Umweltprobleme nicht an Grenzen halt machen.

Noch gilt, dass Südamerika generell über einen großen Reichtum an Naturressourcen verfügt. Dem gegenüber stehen jedoch eine extrem hohe Abbaurate und eine lange Tradition rücksichtsloser Ausbeutung natürlicher Ressourcen, die zum Teil noch heute anhält oder sich in einer auf kurzfristige Gewinne ausgerichteten Unternehmerkultur widerspiegelt. Vor diesem Hintergrund erscheint eine Umorientierung auf nachhaltiges Wirtschaften besonders wichtig. Die Frage ist, ob und wieweit die regionale Wirtschaftsintegration dazu beiträgt.

Die 1995 ins Leben gerufene Umweltarbeitsgruppe des Mercosur Subgrupo de Trabajo No. 6 Medio Ambiente (SGT 6) wird von den Umweltministerien der vier Mitgliedsländer koordiniert. Sie hat offiziell den Auftrag dafür zu sorgen, dass Umweltanliegen und nachhaltige Entwicklung im Integrationsprozess gebührende Beachtung finden. Diese Aufgabenbeschreibung bringt den

\section{Gesellschaftlicher Umgang mit Risiken}

ist das Thema des Informationsdienstes

\section{Ökologisches Wirtschaften 3/05}

Wenn Sie potenzielle Beiträge haben, wenden Sie sich bitte an die Redaktion. transversalen, durch alle Politikbereiche ziehenden Charakter des Umwelt- und Ressourcenschutzes zum Ausdruck, der in letzter Konsequenz eine kohärente Politikgestaltung im Sinne des Leitbildes nachhaltiger Entwicklung verlangt. Ohne Frage ist dieser Anspruch in der realpolitischen Ausgestaltung des Blocks keineswegs reflektiert, dessen Agenda wirtschafts- und handelspolitische Themen beherrschen. Umweltfragen werden in der Regel nachrangig behandelt und oft in einem Atemzug mit nicht-tarifären Handelshemmnissen genannt. Auch wenn die Umweltarbeitsgruppe eine vergleichsweise hohe Kontinuität aufweist, spielte sie zunächst keine proaktive Rolle und befasste sich vorrangig mit wenig konfliktträchtigen Themen.

\section{Rolle der Technischen Zusammenarbeit}

Anfang 2002 startete das erste Vorhaben der Deutschen Technischen Zusammenarbeit mit dem Mercosur: Wettbewerbsfähigkeit und Umwelt - Förderung umweltorientierter Unternehmensführung und sauberer Produktionsverfahren in Klein- und Mittelunternehmen (KMU). Ziel ist die Entwicklung und Umsetzung einer gemeinschaftlichen Förderstrategie zur Stärkung der Wettbewerbsfähigkeit und gleichzeitigen Verbesserung des Umweltverhaltens von KMU. Politischer Träger ist die Umweltarbeitsgruppe des Blocks (SGT 6). Der deutsche Beitrag wird von der Gesellschaft für technische Zusammenarbeit (GTZ) implementiert. Durch eine fachliche Zusammenarbeit zwischen den Ländern und die damit verbundene Stärkung der Umweltarbeitsgruppe soll der Umwelt- und Ressourcenschutz im Mercosur besser positioniert und die Idee ökologisch nachhaltigen Wirtschaftens in relevante ökonomische Initiativen des Regionalblocks hineingetragen werden.

In der ersten Kooperationsphase von 2002 bis 2004 wurden Studien zur Bestimmung des Status Quo sowie gemeinsame Seminare über umweltpolitische Strategien durchgeführt. Als besonders wichtige Projektaufgabe erwiesen sich Austausch und Transfer von Erfahrungen zwischen den Ländern. Basierend auf einer Bestandsaufnahme der im Mercosur vorhandenen Förderansätze und Instrumente für umweltorientiertes Management sowie saubere, effiziente Produktionsverfahren wurden Möglichkeiten zur vermehrten Nutzung und Stärkung des vorhandenen Know-hows aufgezeigt. Für die Projektdynamik erwies es sich als notwendig, nicht nur rein regionale Kooperationsprozesse zu unterstïtzen, sondern auch deren Überset- 
zung auf die nationale Ebene, das heißt die Verzahnung zwischen Anstrengungen des Mercosur und denen seiner Mitgliedsländer. Regionale Integration setzt voraus, dass die beteiligten Länder ihre Hausaufgaben erledigen. So sind neben den oft mühsamen und abstimmungsintensiven Fortschritten bei der gemeinsamen Strategieentwicklung auf Mercosur-Ebene interessante Entwicklungen auf nationaler Ebene zu registrieren.

Beispiele dafür sind die Erarbeitung eines Operationsplans zur Umsetzung der argentinischen Politik für saubere Produktion (Producción Limpia) und die Schaffung von Koordinationsstrukturen oder Runden Tischen zur Förderung ökologischen Wirtschaftens. Auch wenn diese Strukturen erst am Anfang stehen, sind es viel versprechende Ansätze.

\section{- Prioritäre Aktivitäten}

Seit Beginn der zweiten Phase von 2004 bis 2007 konzentriert sich das Vorhaben mit dem Mercosur auf folgende Handlungsfelder:

- Erarbeitung einer gemeinschaftlichen Politik zur Förderung umweltgerechten Wirtschaftens: Gegenwärtig werden Vorschläge in einem Format erarbeitet, das sich als Vorlage für die Entscheidungsorgane des Mercosur eignet.

- Austausch, Kommunikation und Kooperation im Bereich nachhaltigen Wirtschaftens sowohl zwischenstaatlich als auch zwischen öffentlichem und privatem Sektor: Diesem Aktivitätsbereich wird von allen Partnern großer Stellenwert beigemessen. Nach Etablierung von Koordinationsstrukturen in jedem der vier Mitgliedsländer wurde im November 2004 in Brasilien ein öffentlichprivates Dialogforum des Mercosur durchgefuihrt, bei dem auch die nationalen Gremien vertreten waren. Es wurde großer Bedarf für regionale Vernetzung festgestellt. In welcher Form dies allerdings geschehen kann, ist angesichts der finanziellen und organisatorischen Implikationen noch zu klären. Das Thema Wettbewerbsfähigkeit und Umwelt soll künftig auch im Umweltinformationssystem des (SIAM) verankert werden.

- Austausch und Transfer praktischer Erfahrungen mit Konzepten und Instrumenten für umweltorientiertes Management und saubere Produktion. Dieser Punkt umfasst punktuell auch die Förderung modellhafter Umweltmanagementaktivitäten mit definierten Gruppen von Unternehmen unter der Maßgabe, eng mit anderen Initiativen zu kooperieren, bevorzugt vorhandene Methoden und Instrumente zu nutzen sowie kontinuierlich Ergebnisse zu dokumentieren und aus- zutauschen. Von der GTZ wurde das speziell für KMU entwickelte Programm profitables Umweltmanagement (PRUMA) eingeführt (1). Außerdem sollen künftig deutsche und multinationale Unternehmen aktiv eingebunden werden, die mit ihren Erfahrungen und Kapazitäten dazu beitragen können, dass ihre Zulieferer, in vielen Fällen KMU, Anstrengungen für ökologisches Wirtschaften unternehmen und damit Ihre Wettbewerbsfähigkeit verbessern.

\section{- Entwicklungen im Projektumfeld}

Der Start des gemeinsamen Vorhabens mit der GTZ bedeutete für die Umweltarbeitsgruppe des Mercosur die erste wichtige internationale Kooperation und zugleich eine große Herausforderung. Ab Mitte 2003 ist eine Konsolidierung ihrer Arbeit zu verzeichnen, begleitet von generellen Fortschritten im Umwelt- und Ressourcenschutz. Die bedeutendste Neuerung war die Einrichtung eines regelmäßigen Treffens der Umweltminister Argentiniens, Brasiliens, Paraguays und Uruguays als offizielles Organ des Mercosur. Ein wichtiger Schritt ist auch das Mitte 2004 in Kraft getretene Umwelt-Rahmenabkommen des Wirtschaftsblocks. Durch Institutionalisierung des Ministertreffens hat das Umweltthema im MERCOSUR größeres Gewicht bekommen. Dies kommt auch dem TZ-Vorhaben mit der GTZ zugute, das vor allem eine Katalysatorrolle spielt und seine Empfehlungen besser in die politische Diskussion einspeisen kann. Insgesamt ist festzustellen, dass die Kooperation des Mercosur im Umweltbereich sowohl intern als auch auf internationaler Ebene wie zum Beispiel mit der UNEP und in Bezug auf globale Umweltkonventionen wesentlich an Dynamik gewonnen hat. Von Mitgliedern der Umweltarbeitsgruppe wird anerkannt, dass die Erfahrungen mit der deutschen TZ dabei hilfreich waren.

\section{- Herausforderungen}

In Mercosur-Kreisen wird daran erinnert, dass die ursprünglichen Impulse für den Integrationsprozess nicht nur vom Handel sondern auch von der Überlegung ausgingen, dass man durch Zusammenarbeit Schwächen in den Produktionsstrukturen der einzelnen Länder ausgleichen und Stärken nutzen könne, um gemeinsam eine bessere Weltmarktposition zu erreichen. Das Konzept industrieller Ergänzung, das in der realen Mercosur-Entwicklung zunächst keine Rolle spielte, bildete den Ausgangspunkt eines aktuellen Programms zur Stärkung der wirtschaftlichen
Integration entlang definierter Wertschöpfungsketten (Programa Foros de Competitividad). Diese von der Industriearbeitsgruppe (SGT 7) eingebrachte Initiative genießt die Unterstiutzung der obersten Organe des Mercosur bis hin zu den Staatspräsidenten. Als Pilotfall, dem andere folgen sollen, wurde am 31. März 2003 in Asunción das erste Forum für Wettbewerbsfähigkeit in der Kette Holz - Möbel ins Leben gerufen.

Obwohl auch hier zunächst weder Umwelt- noch Nachhaltigkeitsaspekte explizite Berücksichtigung fanden, gibt es auch dank des TZ-Vorhabens Wettbewerbsfähigkeit und Umwelt mittlerweile gute Chancen, die Dimension Umwelt bei der Umsetzung des Programms einzubeziehen. Die Umweltrelevanz des Rohstoffes Holz ist einfach zu offensichtlich und viele Exportmärkte fragen Produkte aus nachhaltiger Waldwirtschaft nach. Das Vorhaben wird einen Beitrag zu einer integrierten Herangehensweise leisten, die sich nicht auf einzelne Unternehmen oder Sektoren beschränkt, sondern alle Glieder der Wertschöpfungskette in Betracht zieht. Zwar bestehen heute Instrumente wie die Produktzertifizierung oder die Lebenszyklusanalyse, die eine Betrachtung der gesamten Kette von der Primärproduktion über Verarbeitung, Handel und Konsum bis hin zur Post-Konsum-Phase beinhalten. Der Wertschöpfungskettenansatz geht jedoch darüber hinaus: Er basiert auf einer integrierten Sichtweise der Prozesse entlang der gesamten Wertschöpfungskette unter Einbeziehung aller Akteure.

Das Prinzip der Ökoeffizienz, mehr Wertschöpfung bei weniger Ressourcenverbrauch, ist mit den Prozessen und Programmen zur regionalen Integration im Mercosur voll kompatibel. Es bringt langfristig hohen wirtschaftlichen Nutzen, erfordert jedoch eine Abkehr von der kurzfristig orientierten Unternehmerkultur und der Ausbeutung von Naturvorräten hin zu einer nachhaltigen, wissensbasierten Produktionsweise.

\section{Anmerkung}

(1) Für weitere Informationen www.gtz.de/p3u

\section{Der Autor}

Dr. Detlef Schreiber ist Senior-Berater der Deutschen Gesellschaft für Technische Zusammenarbeit (GTZ).

Kontakt: Proyecto Competitvidad y Medio Ambiente Mercosur-Alemania, c/o Diname, Rincón 422, 11000 Montevideo, Uruguay. Tel.: 00598-29170710; E-Mail: detlef.schreiber@gtz.de 
(c) 20I0 Authors; licensee IÖW and oekom verlag. This is an article distributed under the terms of the Creative Commons Attribution Non-Commercial No Derivates License (http://creativecommons.org/licenses/by-nc-nd/3.o/), which permits unrestricted use, distribution, and reproduction in any medium, provided the original work is properly cited. 